\title{
Steroid receptor coactivator 3 inhibits hepatitis B virus gene expression through activating Akt signaling to prevent HNF4a nuclear translocation
}

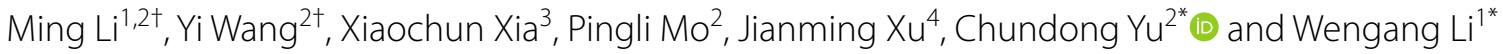

\begin{abstract}
Background: Chronic hepatitis B virus (HBV) infection is one of the most serious global public health problems. The role of steroid receptor coactivator 3 (SRC-3) in HBV biosynthesis is unknown. The aim of this study is to investigate the function of SRC-3 in regulating HBV biosynthesis both in vitro and in vivo and to identify the underlying mechanism.

Results: In this study, we found that knockdown of SRC-3 could increase the levels of HBV mRNA and HBV proteins HBsAg and HBeAg in human liver cancer cell line HepG2 transfected with pHBV1.3 plasmids. In contrast, enforced expression of SRC-3 in SRC-3-knockdown HepG2 cells reduced the levels of HBV mRNA and HBV proteins HBsAg and HBeAg. Knockdown of SRC-3 dampened the Akt signaling, which has been shown to play a negative role in HBV transcription. Ectopic expression of constitutively activated Akt impaired the enhancement of HBV transcription by SRC-3 knockdown, indicating that SRC-3 inhibits HBV transcription by enhancing Akt signaling. Both SRC-3 and constitutively activated Akt could inhibit hepatocyte nuclear factor $4 a$ (HNF4a)-mediated upregulation of HBV core promoter activity by preventing HNF4a nuclear translocation. Consistent with the in vitro results, in an in vivo chronic HBV replication mouse model developed by hydrodynamic injection of pHBV1.3 plasmids into mouse tail vein, enforced expression of SRC-3 in mouse liver reduced the levels of HBV mRNA in the liver and HBV antigens in serum, whereas knockout of SRC-3 in mouse increased the levels of HBV mRNA in the liver and HBV antigens in the serum.
\end{abstract}

Conclusion: Our study suggests that SRC-3 inhibits HBV gene expression by activating Akt signaling to prevent HNF4a nuclear translocation.

Keywords: SRC-3, Hepatitis B virus, Akt, HNF4a

\section{Introduction}

Hepatitis B virus (HBV) infection is the most common chronic viral infection in the world. Despite significant improvement in the management of HBV, it is still a global public health problem [1]. About 2 billion

\footnotetext{
*Correspondence: cdyu@xmu.edu.cn; Iwg11861@163.com

${ }^{\dagger}$ Ming Li and Yi Wang contributed equally to this work

${ }^{1}$ Department of Hepatobiliary and Pancreatic \& Organ Transplantation Surgery, Xiang'an Hospital of Xiamen University, School of Medicine,

Xiamen University, Xiamen 361012, Fujian, China

${ }^{2}$ State Key Laboratory of Cellular Stress Biology, School of Life Sciences,

Xiamen University, Xiamen 361012, Fujian, China

Full list of author information is available at the end of the article
}

people were infected with HBV worldwide and more than 350 millions have become chronic infection [2, 3]. Chronic HBV infection greatly increases the risk of chronic liver disease, including hepatitis, fibrosis, cirrhosis, and liver cancer [3].

Hepatitis B virus is a small enveloped hepatotropic DNA virus that belongs to the Hepadnaviridae family and its life cycle involves both DNA and RNA intermediates [3]. HBV genome $(3.2 \mathrm{~kb})$ is circular partial duplex DNA, also calling relaxed circular (rc) DNA. After HBV entering hepatocyte, rcDNA genome is released into the nucleus. In hepatocyte nucleus, cellular enzymes 
convert the rcDNA to a covalently closed circular DNA (ccc DNA) [4, 5]. cccDNA is a key component in the HBV life cycle, since it is the template for pregenomic/precore RNAs (3.5-kb) and subgenomic RNAs (2.4, 2.1, 0.7-kb) [4, 5]. HBV genome cccDNA has widely overlapping open reading frames (ORFs) and regulatory elements overlap with coding sequence, therefore a $3.2 \mathrm{~kb} \mathrm{HBV}$ genome has 5 transcriptions depending on different promoter regions (both 3.5, 2.4, 2.1 and 0.7-kb) and some transcriptions have multiple ORFs depending on different translation regions [5]. The pregenomic RNA (pgRNA) serves as mRNAs for generating hepatitis B core antigen $(\mathrm{HBcAg})$ and polymerase/reverse transcriptase protein and is the template of reverse transcription to produce the HBV DNA [5]. HBcAg protein constitutes viral capside and viral DNA genome and virally encoded polymerase were inside of the capside [5]. The Precore RNA (preRNA) serves as an mRNA for generating the secreted hepatitis $\mathrm{B}$ e antigen ( $\mathrm{HBeAg}$ ) and the function of $\mathrm{HBeAg}$ may be involved in immune toleration [5]. The $2.4 \mathrm{~kb}$ subgenomic RNA serves as an mRNA for generating large HBsAg (hepatitis B surface antigen) protein and $2.1 \mathrm{~kb}$ subgenomic RNA serves as an mRNA for generating middle and small HBsAg [5]. The viral capside is surrounded by a lipid envelope containing $\mathrm{HBsAg}$. HBsAg and $\mathrm{HBeAg}$ are viral markers detected in serum [5]. The 0.7-kb subgenomic RNA serves as an mRNA for generating $\mathrm{HBx}$ protein which contributes to HBV infection and oncogenic potential.

HBV replicates by reverse transcription of $3.5-\mathrm{kb}$ pgRNA, therefore the level of this transcript is a primary determinant of viral biosynthesis [4]. Extensive studies demonstrate that a number of ubiquitous transcription factors and liver-enriched transcription factors/ nuclear receptors bind to the HBV promoter/enhancer regions and regulate the activity of these regulator elements, and in turn control the transcription of HBV gene [4]. Hepatocyte nuclear factor $4 \alpha(\mathrm{HNF} 4 \alpha)$ is a member of the nuclear receptor family and regulates the expression of $44 \%$ of hepatocyte-specific genes [6]. Overexpression of HNF4 $\alpha$ increases the levels of HBV mRNA in hepatoma cell lines and non-hepatic cell lines [7-9]. HNF $4 \alpha$ increases the synthesis of HBV pregenomic RNA by activating HBV promoter in hepatoma cell huh-7 [10]. Conditional depletion of HNF4 $\alpha$ in the liver decreases the HBV transcription and replication in HBV transgenic mouse model of chronic infection [11]. These results suggest that HNF4 $\alpha$ is a major regulator of pregenomic RNA transcription and HBV replication, consequently determining the viral biosynthesis. The phosphatidylinositol 3-kinase (PI3K)-protein kinase B/Akt signaling pathway plays crucial role in cell proliferation, differentiation, and survival [12]. Activation of Akt inhibits HBV transcription and replication $[13,14]$. Akt acts at HNF4 $\alpha$ to decrease HBV transcription [14].

Steroid receptor coactivator 3 (SRC-3/ACTR/AIB-1/ pCIP/TRAM-1), is a member of p160 coactivator family. SRC-3 not only interacts with nuclear hormone receptors but also interacts with other transcription factors to enhance their downstream target gene transcription [15]. SRC-3 is overexpressed in human hepatocellular carcinoma $(\mathrm{HCC})$ and promotes tumor progression by enhancing Akt signaling $[15,16]$. Our previous study has demonstrated that HBx protein, a regulator of HBV replication, stabilizes SRC-3 protein and cooperates with it to promote human HCC cell invasiveness [17], indicating that there exist crosstalk between SRC-3 and HBV in the liver. In this study, we investigate the role of SRC-3 in regulating HBV biosynthesis in vitro and in vivo and identify the underlying mechanism.

\section{Materials and methods \\ Animals and cell cultures}

WT and SRC- $3^{-/-}$male mice (6-8 weeks old) were used for hydrodynamic injection experiments. All animal protocols were approved by Animal Care and Use Committee of Xiamen University. HepG2 cells were cultured in DMEM medium supplemented with $10 \%$ FBS.

\section{Plasmids and transfection}

pGL3-EnII/Cp-luc plasmid was constructed in our laboratory. HBV Enhancer II/core promoter element (1399$1890 \mathrm{nt}$ ) was cloned into pGL3-basic vector expressing firefly luciferase to generate pGL3-EnII/Cp-luc. Constitutively active Akt expression plasmid (Akt1-T308D/ S473D) was provided by Dr. Jianming Xu (Baylor College of Medicine, USA). Transfection was performed at the cell-density of $60-80 \%$ per well using Lipofectamine 2000 according to the manufacturer's instruction (Invitrogen).

\section{Measuring of the levels $\mathrm{HBsAg}$ and $\mathrm{HBeAg}$ proteins}

The protein levels of HBsAg and HBeAg in culture media or animal serum were measured by ELISA kit for HBsAg (Cat No. 05.02.01.012; InTec Products, China) and HBeAg (Cat No. 05.02.03.001; InTec Products, China), respectively. Culture media were collected for centrifugation at $13,000 \times g$ for $10 \mathrm{~min}$ to remove cell debris. The blood freshly collected was placed at room temperature for $1 \mathrm{~h}$ to clot, followed by centrifugation at $5500 \times g$ for 5 min to harvest the serum.

\section{Western blot analysis}

Western blotting analysis was performed as previously described [16]. The antibody against SRC-3 (Cat No. 2126S; CST, USA), p-AKT (Cat No. 4060S; CST, USA), 
AKT (Cat No. 9272S; CST, USA), Tublin (Cat No. 2148S; CST, USA), GFP (Cat No. 2956T; CST, USA) and PARP (Cat No. 9532T; CST, USA) were purchase from Cell Signal Technology. The antibody against human HNF4 $\alpha$ (Cat No. ab92378; Abcam, UK) and mouse HNF4 $\alpha$ (Cat No. ab41898; Abcam, UK) was purchase from abcam. The $\beta$-actin antibody (Cat No. A5441; Sigma Aldrich, USA) was purchase from Sigma Aldrich.

\section{Quantitative real-time PCR (qPCR)}

Total RNA was isolated with Trizol reagent (Invitrogen) according to the manufacturer's instructions. The cDNA was synthesized from $2 \mu \mathrm{g}$ of total RNA using MMLV transcriptase (ToYoBo, Shanghai, China) with random primers. Real-time PCRs were performed using SYBR Premix ExTaq (TaKaRa, Dalian, China). Specific primers used to detect HBV total mRNAs by real-time PCR were as follows: forward primer, 5'-ACGTCCTTTGTTTAC GTCCCGT-3', reverse primer, 5'-CCCAACTCCTCC CAGTCCTTAA- $3^{\prime}$ [18]. All the other real-time primers are listed as follows: IGF1 forward primer, $5^{\prime}$-TTT ATTTCAACAAGCCCACA-3', IGF1 reverse primer, 5'-TCTCCAGCCTCCTTAGATC-3'; IRS1 forward primer, 5'-CTTCGGTGTCTGGTTCCC-3', IRS1 reverse primer, 5'-ATAGTTGCTTAGCTCCTCCTCA-3'; IRS2 forward primer, 5'-GGCTTGGTCGGTTGTCCTGG-3', IRS2 reverse primer, 5'-CCTCACGTCGATGGCGAT GTAG-3'.

\section{Luciferase reporter assay}

pGL3-EnII/Cp-luc plasmid was tranfected into HepG2 cells together with pCR3.1-Rluc. Cells were assayed at $48 \mathrm{~h}$ post-transfection for luciferase activity and normalized to Renilla luciferase activity using a dual luciferase reporter assay system (Promega, Madison, WI, USA).

\section{Hydrodynamic injection (HI) mouse model}

To test HBV replication in vivo, mice were subjected to hydrodynamic injection with 5 mice per group. The HI method has been described previously [19]. Briefly, WT and SRC- $3^{-1-}$ male mice were injected with $10 \mu \mathrm{g}$ pHBV1.3 plasmid together with $1 \mu \mathrm{g}$ pPCR3.1-Renillaluciferase plasmid DNA in PBS with a volume of $8 \%$ $(\mathrm{w} / \mathrm{v})$ bodyweight via tail vein within $5-8 \mathrm{~s}$. WT male mice were injected with $10 \mu \mathrm{g}$ pHBV1.3 plasmid and $1 \mu \mathrm{g}$ PCR3.1-Renilla-luciferase plasmid together with $10 \mu \mathrm{g}$ pPCR3.1-SRC-3 plasmid or the same molar control plasmid DNA in PBS with a volume of $8 \%(\mathrm{w} / \mathrm{v})$ body weight via tail vein within $5-8 \mathrm{~s}$. The efficiency of transfection was normalized to the activity of Renilla-luciferase. To assess the transfection efficiency of HI mouse model in this study, $10 \mu \mathrm{g}$ PCR3.1-GFP plasmids were injected into mouse and liver cells expressing GFP gene were identified by immunochemistry. There were approximately $25 \%$ of cells expressing GFP gene in mouse liver section (Additional file 1: Figure S1).

\section{Statistical analysis}

Statistical analysis was performed using SPSS 17.0 for Windows. Differences between groups were evaluated with t-test. $\mathrm{p}$ value $<0.05$ was considered significant.

\section{Results}

SRC-3 inhibits HBV biosynthesis in HepG2 cells

To investigate the role of SRC-3 in HBV biosynthesis, we established stable SRC-3-knockdown HepG2 cells using pSuper plasmid containing shRNA against SRC-3 or scramble control shRNA as described previously [16]. The shSRC-3, but not scramble shRNA (shCtrl) efficiently knocked down the expression of SRC-3 in HepG2 cells (Fig. 1a). The HBV biosynthesis model was established by transfecting pHBV1.3 plasmids into control and SRC3-knockdown HepG2 cells, respectively. Downregulation of SRC-3 increased HBV mRNA levels in cells (Fig. 1b), as well as the protein levels of HBsAg and HBeAg in culture media (Fig. 1c), indicating that SRC-3 could negatively regulate $\mathrm{HBV}$ transcription and viral protein production. Furthermore, we performed SRC-3 rescue experiment to confirm the role of SRC-3 in HBV biosynthesis (Fig. 1d). Stable SRC-3-knockdown cells were transiently transfected with SRC-3 expression plasmids or control plasmids together with pHBV1.3 plasmids for $48 \mathrm{~h}$, the protein levels of SRC-3 were detected by Western blot, the mRNA levels of HBV were quantified by real-time PCR, and the protein levels of HBsAg and $\mathrm{HBeAg}$ were measured by ELISA. As expected, the levels of HBV mRNA as well as HBsAg and $\mathrm{HBeAg}$ proteins were downregulated when overexpressing SRC-3 in SRC3-knockdown cells (Fig. 1e, f). These results suggest that SRC-3 inhibits HBV gene transcription and viral protein production, consequently reducing HBV biosynthesis in HepG2 cells.

\section{SRC-3 activates Akt to inhibit HBV biosynthesis}

It has been demonstrated that activation of Akt inhibits HBV gene transcription and replication [13, 14]. Previous study has demonstrated that SRC-3 up-regulates the transcription of multiple genes in insulin-like growth factor (IGF)/AKT signaling pathway [such as IGF1, insulin receptor substrate (IRS) 1 and IRS2] to activate AKT signaling [20]. Consistent with previous studies [16, 20], we found that downregulation of SRC-3 significantly reduced IGF1, IRS1, and IRS2 mRNA expression (Additional file 2: Figure S2) and the level of phosphorylated Akt (activated form of Akt) was significantly decreased in 

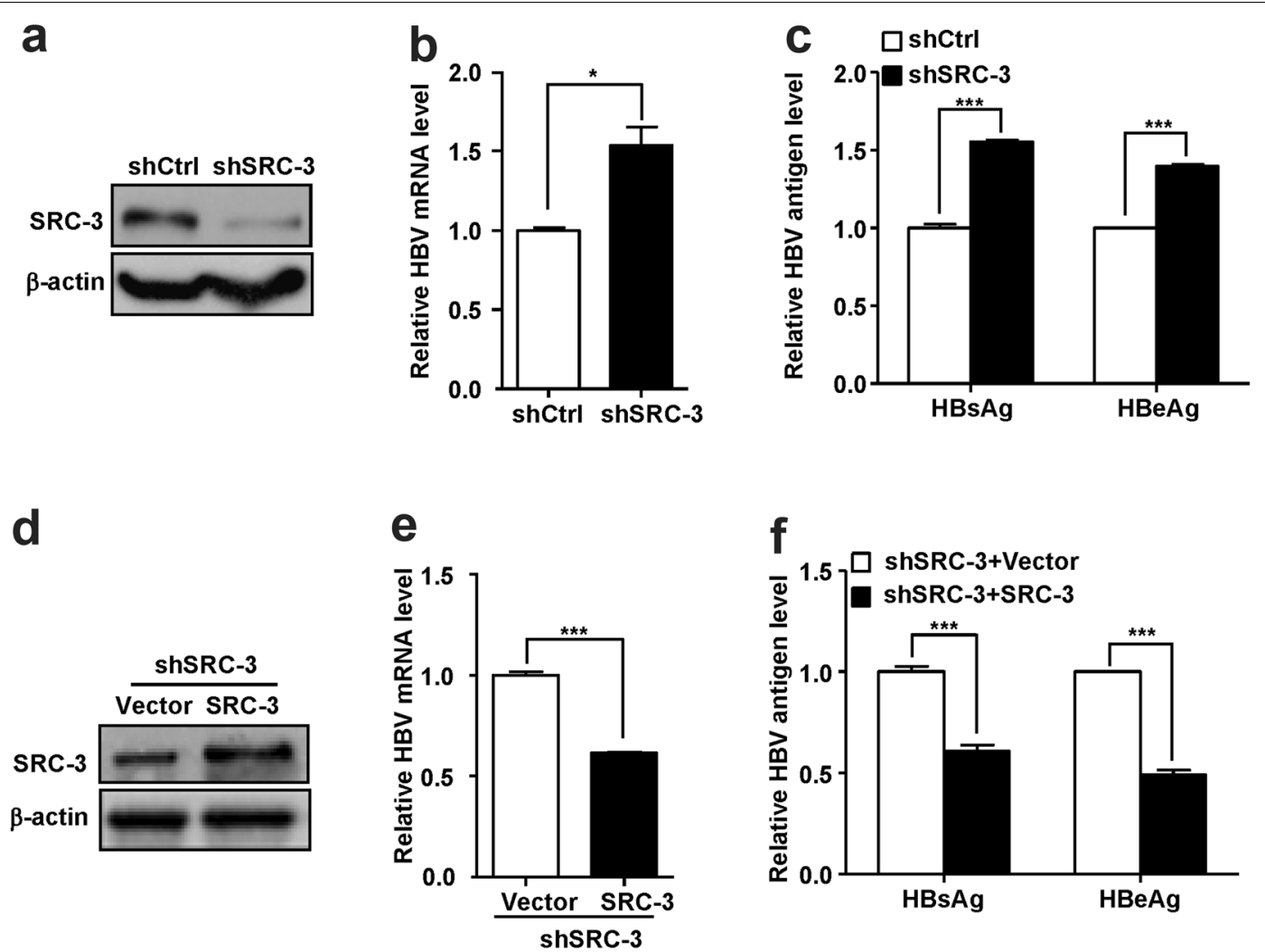

Fig. 1 SRC-3 inhibits HBV biosynthesis in HepG2 cells. a-c Knockdown of SRC-3 increased the mRNA levels of HBV and the protein levels of HBsAg and HBeAg. Stable SRC-3-knockdown and control HepG2 cells were transfected with pHBV1.3 plasmids, respectively. The SRC-3 protein was tested by western blot (a), the mRNA level of HBV was quantified by real-time PCR (b), and the protein of HBsAg and HBeAg was measured by ELISA (c). d-f Ectopic expression of SRC-3 in SRC-3-knockdown HepG2 cells reduced the mRNA levels of HBV and the protein levels of HBsAg and HBeAg. Stable SRC-3-knockdown HepG2 cells were transfected with SRC-3/pHBV1.3 plasmids or control/pHBV1.3 plasmids for 48 h. The protein levels of SRC-3 were detected by western blot (d), the mRNA level of HBV was quantified by real-time PCR (e), and the protein of HBsAg and HBeAg was measured by ELISA (f). All experiments were repeated at least 3 times independently. ${ }^{*} p<0.05,{ }^{* * *} p<0.001$

SRC-3-knockdown cells (Fig. 2a), implicating that SRC-3 may inhibit HBV biosynthesis by activating Akt. To test this hypothesis, we rescued Akt activity in SRC-3-knockdown cells by transfecting constitutively active Akt (CaAkt) expression plasmids (Fig. 2b), and then examined HBV biosynthesis. The results showed that the levels of HBV mRNA (Fig. 2c) and HBsAg and HBeAg proteins (Fig. 2d) were significantly decreased after transfection of Ca-Akt expression plasmids into SRC-3-knockdown cells. Collectively, these results suggest that SRC-3 inhibits HBV biosynthesis by enhancing Akt signaling.

\section{SRC-3 inhibits $\mathrm{HBV}$ promoter activity through regulating Akt/HNF4a signal axis}

It has been reported that hepatocyte-specific transcription factor HNF4 $\alpha$ increases the synthesis of HBV pregenomic RNA by activating HBV core promoter [7, 10], but the positive effect of HNF4 $\alpha$ on HBV RNA synthesis could be attenuated by Akt signaling [14]. To determine whether SRC-3 could inhibit HNF4 $\alpha$-induced HBV biosynthesis, we cloned HBV Enhancer II/core promoter element (1399-1890 nt) into pGL3-basic firefly luciferase reporter plasmids to generate pGL3-EnII/Cp-luc, and then transfected pGL3-EnII/Cp-luc plasmids along with control plasmids, SRC-3 expression plasmids, or constitutively active Akt (Ca-Akt) expression plasmids into HepG2 cells, respectively. As shown in Fig. 3a, HNF4a enhanced the HBV enhancer II/core promoter activity as expected, but overexpression of SRC-3 or Ca-Akt inhibited $\mathrm{HNF} 4 \alpha$-induced $\mathrm{HBV}$-core promoter activity. In contrast, knockdown of SRC-3, which reduced Akt activity (Fig. 3c), increased HBV-core promoter activity (Fig. 3b). Restoration of Akt activity in SRC-3-knockdown cells by transfecting $\mathrm{Ca}$-Akt expression plasmids repressed HBV-core promoter activity (Fig. 3b). These results suggest that SRC-3 inhibits HNF4 $\alpha$-induced HBV-core promoter activity by activating Akt signaling. Knockdown of SRC-3 had no effect on HNF4 $\alpha$ expression, but promoted the nuclear translocation of HNF4 $\alpha$ (Fig. 3c). Restoration of Akt activity in SRC-3-knockdown cells inhibited the 

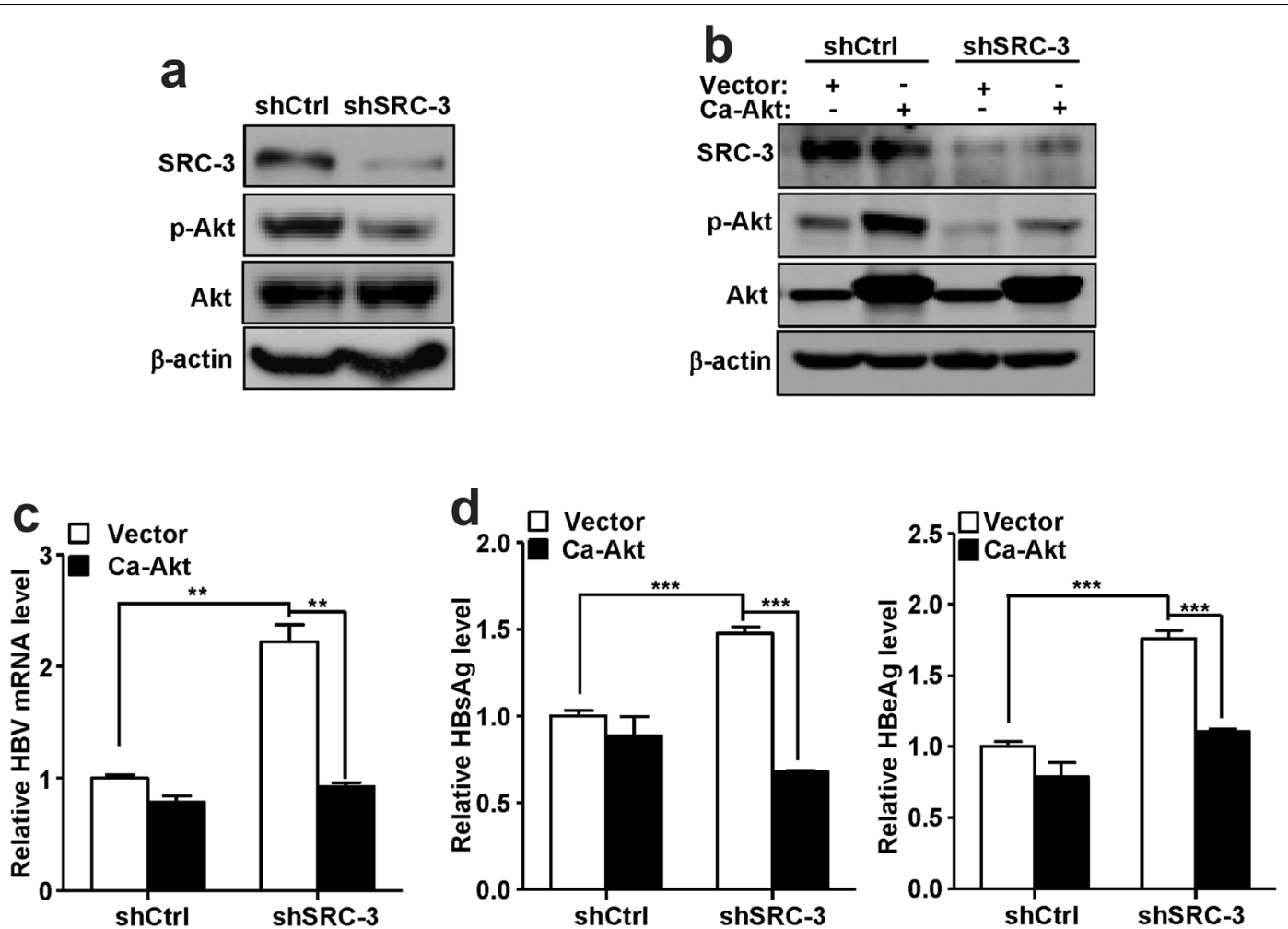

Fig. 2 SRC-3 activates Akt signaling to inhibit HBV gene expression. a Knockdown of SRC-3 decreased phosph-Akt expression in HepG2 cells. The expression of $\mathrm{p}$-Akt and t-Akt were analyzed by western bolt. $\beta$-actin was served as a loading control. b-d Ectopic expression of constitutively active Akt (CA-Akt) reduced the mRNA levels of HBV and the protein levels of HBsAg and HBeAg. SRC-3-knockdown or control HepG2 cells were transfected with CA-Akt and pHBV1.3 plasmids for $48 \mathrm{~h}$, and then the protein levels of SRC-3, p-Akt and t-Akt were detected by western blot (b), the mRNA level of HBV was quantified by real-time PCR (e), and the protein levels of HBsAg and HBeAg was measured by ELISA (d). All experiments were repeated at least 3 times independently. ${ }^{* *} p<0.01,{ }^{* *} p<0.001$

nuclear translocation of HNF4 $\alpha$ (Fig. 3c). These results suggest that SRC-3 inhibits HBV promoter activity by preventing $H N F 4 \alpha$ nuclear translocation via activation of Akt signaling.

\section{SRC-3 inhibits HBV biosynthesis in vivo}

To detect the effect of SRC-3 on HBV biosynthesis in vivo, hydrodynamic injection (HI) mouse model was adopted to co-express HBV and SRC-3 in the liver. The mRNA levels of SRC-3 in the liver were significantly upregulated after intravenous injection of SRC-3 expression plasmids (Fig. 4a), whereas the HBV mRNA levels in the liver and the protein levels of HBsAg and HBeAg in the serum were decreased (Fig. 4b, c). Furthermore, SRC $-3^{-1-}$ mice were used to determine the effect of SRC-3 deficiency on HBV biosynthesis by intravenously injecting pHBV1.3 plasmids. SRC-3 deficiency increased the mRNA levels of HBV in the liver (Fig. 4d), as well as the proteins levels of $\mathrm{HBsAg}$ and $\mathrm{HBeAg}$ in the serum (Fig. 4e). These results suggest that SRC-3 indeed can inhibit HBV transcription and viral protein production in vivo. In consistent with in vitro data, SRC-3 deficiency decreased the levels of phosphorylated Akt in the liver (Fig. 4f), but promoted the nuclear translocation of HNF4 $\alpha$ (Fig. 4g), indicating that SRC-3 inhibited HBV biosynthesis in vivo by activating Akt signaling to prevent HNF4 $\alpha$ nuclear translocation.

\section{Discussion}

Our previous study showed that SRC-3 was overexpressed in human HCC specimens and promoted HCC progression, and $\mathrm{HBV} X$ protein $(\mathrm{HBx})$, a regulator of HBV replication, interacted and stabilized SRC-3 protein $[16,17]$. However, the role of SRC-3 in HBV biosynthesis remains unclear. In this study, our results showed that knockdown of SRC-3 increased the expression of HBsAg and $\mathrm{HBeAg}$ proteins in cell media and HBV mRNA in cells; consistently, knockout of SRC-3 in mouse increased the expression of $\mathrm{HBsAg}$ and $\mathrm{HBeAg}$ proteins in the serum and HBV mRNA in the liver. In contrast, overexpression of SRC-3 decreased the expression of HBsAg, $\mathrm{HBeAg}$ proteins and $\mathrm{HBV}$ mRNA in vitro and in vivo. 


\section{a}

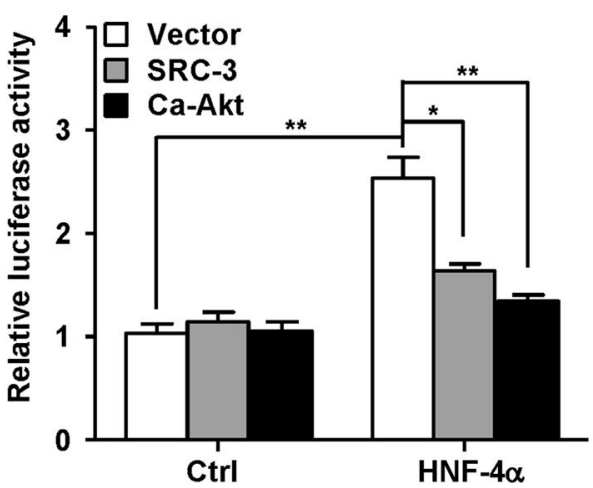

b HBV core-promoter reporter

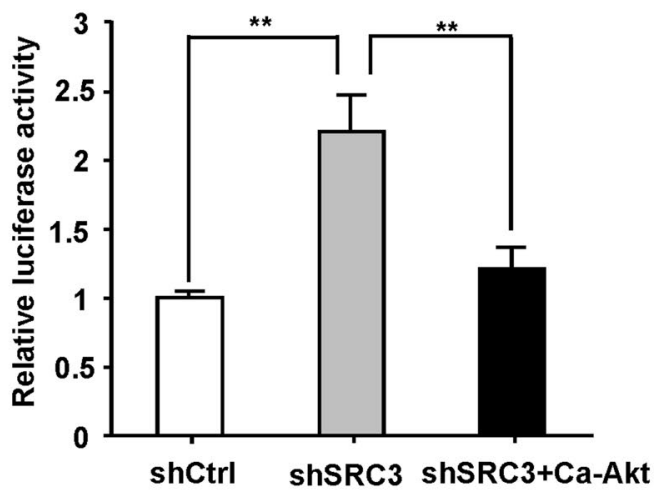

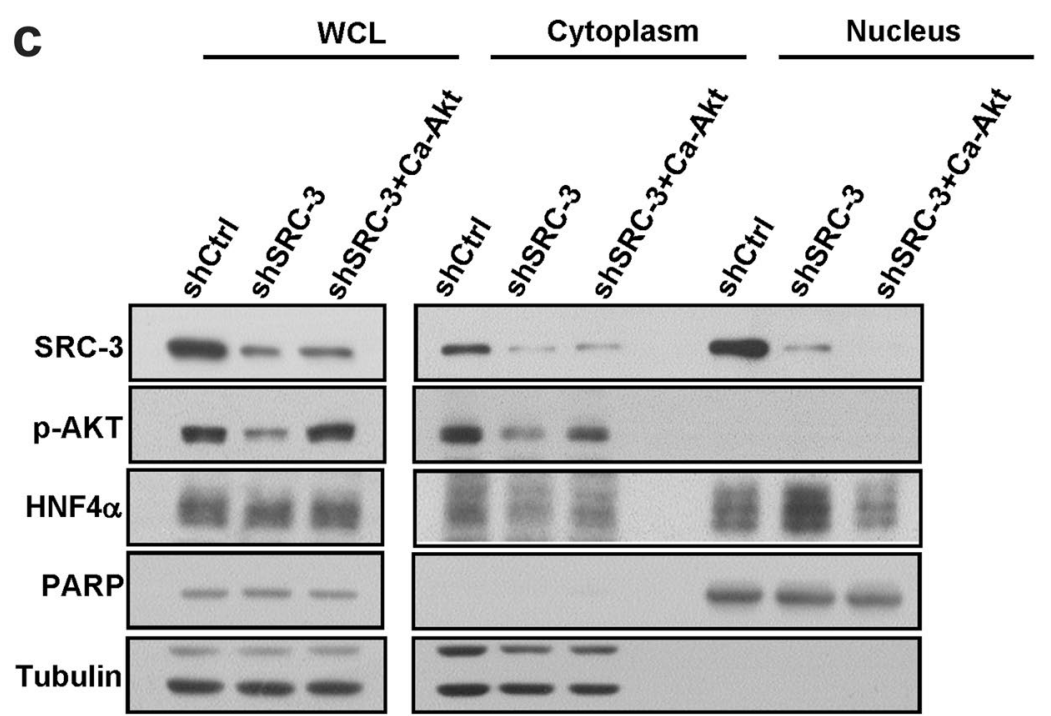

Fig. 3 SRC-3 inhibits HNF4a-induced HBV core-promoter activity. a Ectopic expression of SRC-3 or CA-Akt inhibited HNF4a-induced HBV core-promoter activity in HepG2 cells. b Transfection of CA-Akt into SRC-3-knockdown cells inhibited HBV core promoter activity. c Transfection of CA-Akt into SRC-3-knockdown cells prevented the nuclear translocation of HNF4a. Control constructs were transfected into control cell or SRC-3-knockdown cells and CA-Akt were transfected into SRC-3-knockdown cells for $48 \mathrm{~h}$, then cell fractionation was carried out and fractions were analyzed by western blot. All experiments were repeated at least 3 times independently. ${ }^{*} p<0.05,{ }^{*} p<0.01$

Our study demonstrates that SRC-3 inhibits HBV gene transcription and viral protein production.

The level of HBV gene transcription determines the HBV gene expression and HBV biosynthesis [4]. Activation of PI3 K/Akt frequently happens in numerous types of human cancers and promotes tumor progression [21, 22]. It has been shown that activation of Akt is one of the most consistent characteristic of HBV-induced HCC in a colligated microarray assay [23]. However, activation of Akt inhibits HBV gene transcription and consequently decreases HBV biosynthesis [13, 14]. Our results showed that knockdown of SRC-3 reduced phosphorylated-Akt expression, but increased HBV gene transcription and protein expression; restoration of Akt activity in SRC3-knockdown cells repressed HBV gene transcription and protein expression. These results indicate that SRC3-mediated decrease of HBV biosynthesis is linked to the increase of Akt activity.

A number of liver-enriched transcription factors/ nuclear receptors determines HBV gene transcription 

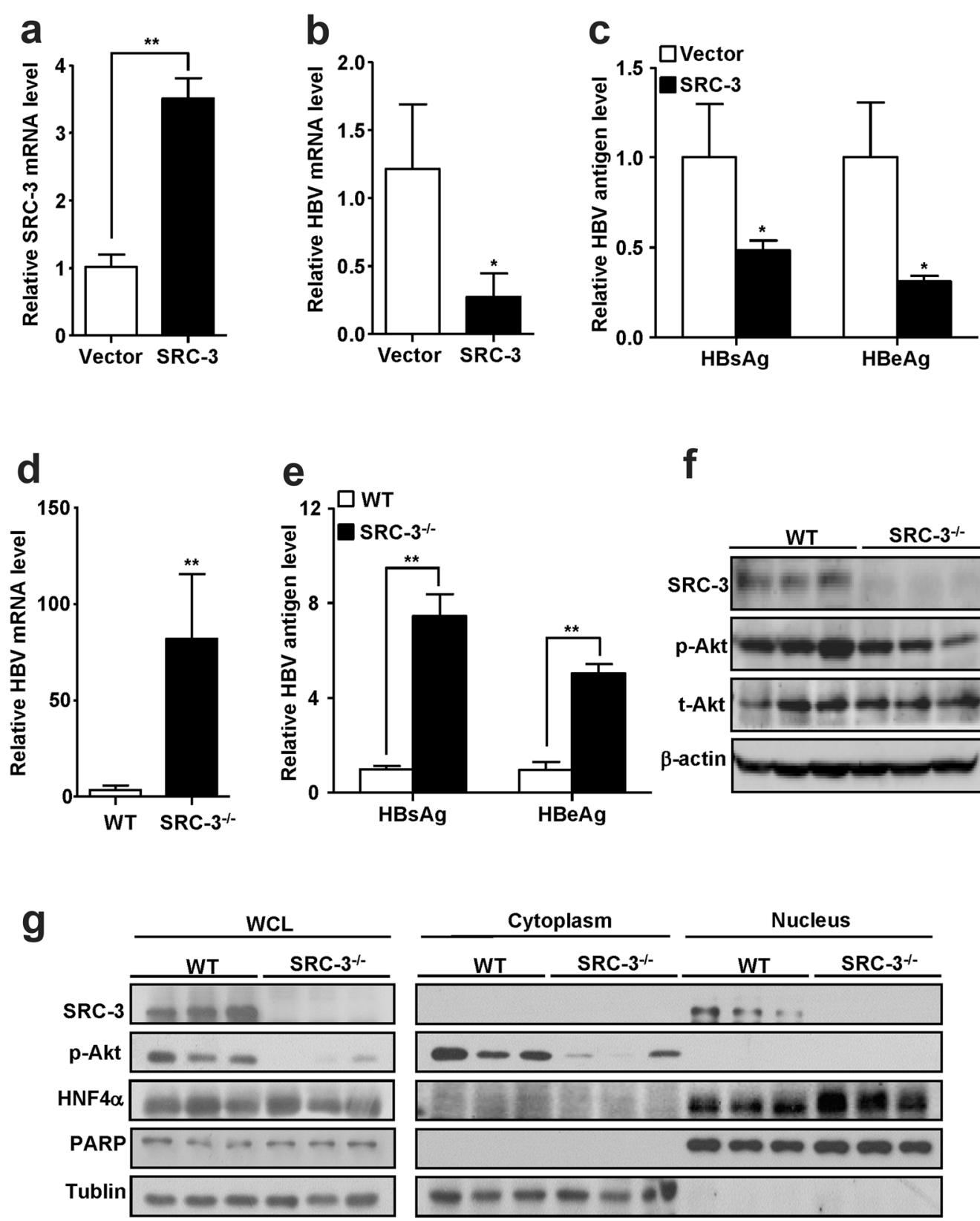

Fig. 4 SRC-3 inhibits HBV gene expression in vivo in a hydrodynamic injection (HI) mouse model. a-c Ectopic expression of SRC-3 in mouse liver reduced the mRNA levels of HBV in the liver and the protein levels of HBsAg and HBeAg in the serum. The control plasmids and SRC-3 expression plasmids, together with pHBV1.3, were transduced into mice by tail vein hydrodynamic injection, respectively. The transfection efficiency was normalized to Renilla luciferase. The mRNA levels of SRC-3 (a) and HBV (b) were quantified by real-time PCR. The serum concentrations of HBsAg and HBeAg proteins were measured by ELISA (c). d , e Knockout of SRC-3 in mouse increased the mRNA levels of HBV in the liver (d) and the protein levels of HBsAg and HBeAg in the serum (e). Wild-type (WT) and SRC-3 $3^{-/}$mice were injected with pHBV1.3 plasmids by tail vein hydrodynamic injection, respectively. The serum concentrations of HBsAg and HBeAg were measured by ELISA; and the mRNA level of HBV was quantified by real-time PCR. $\mathbf{f}$ Knockout of SRC-3 in mouse reduced the levels of $\mathrm{p}$-Akt in the liver. The protein levels of SRC-3, p-Akt, and t-Akt in WT and SRC-3-1liver tissues were detected by western bolt. $\mathbf{g}$ SRC-3 deficiency promoted the nuclear translocation of HNF4a. Mouse liver fractionation was carried out and fractions were analyzed by western blot. All experiments were repeated twice independently. ${ }^{*} p<0.05,{ }^{* *} p<0.01$ 


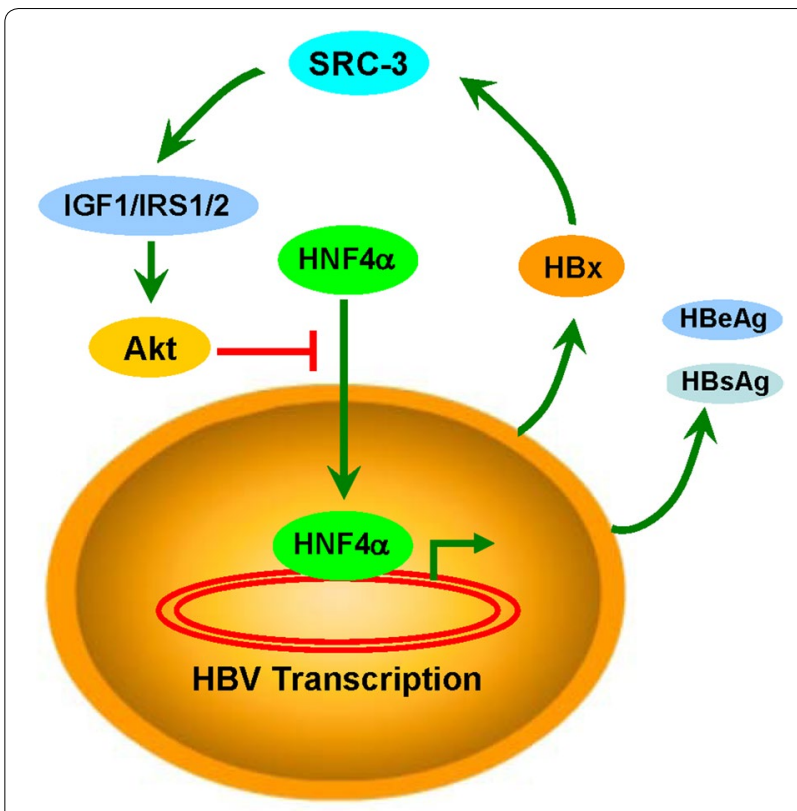

Fig. 5 A schematic diagram depicting the molecular mechanism by which SRC-3 inhibits HBV gene expression. SRC-3 inhibits HBV gene expression by activation of Akt signaling via inducing the expression of IGF1, IRS1, and IRS2 to prevent HNF4a nuclear translocation; HBV gene expression results in $\mathrm{HBx}$ protein production to stabilize SRC-3 protein, leading to a feedback inhibition of HBV gene expression

level and represents a crucial determinant of HBV liver tropism [4]. It has been shown that HNF4 $\alpha$ promotes HBV gene transcription and consequently increases HBV replication [7-10]. In addition, activation of Akt can phosphorylate HNF4 $\alpha$ and result in the translocation of HNF $4 \alpha$ out of the nucleus [24]. Therefore, HNF4 $\alpha$ acts at downstream of Akt signaling for regulation of HBV transcription [14]. Our results showed that both SRC-3 and Akt inhibited HNF4 $\alpha$-induced HBV-enhancer II/core promoter activity; restoration of AKT activity in SRC3-knockdown cells reduced the nuclear translocation of HNF4 $\alpha$ protein as well as HBV-core promoter activity (Fig. 3b, c). These results indicate that SRC-3 inhibits HBV gene transcription at least in part through activating Akt to inhibit HNF4 $\alpha$ nuclear translocation.

We previously reported that $\mathrm{HBx}$ could interact with SRC-3 protein to prevent SRC-3 protein degradation [16, 17]. Based on our previous and current studies, we propose a working model to depict the molecular mechanism by which SRC-3 inhibits HBV gene expression: SRC-3 inhibits HBV gene expression by activation of Akt signaling via inducing the expression of IGF1, IRS1, and IRS2 to prevent HNF4 $\alpha$ nuclear translocation; HBV gene expression results in $\mathrm{HBx}$ protein production to stabilize SRC-3 protein, leading to a feedback inhibition of HBV gene expression (Fig. 5). Taken together, our study demonstrates an important role of SRC-3 in the control of HBV.

\section{Additional files}

Additional file 1: Figure S1. Histochemical analysis of GFP gene expression in mouse liver. $10 \mu \mathrm{g}$ PPCR3. 1-GFP plasmids were injected into mouse and the animal was killed $8 \mathrm{~h}$ after injection. Mouse liver cells expressing GFP gene were identified by immunochemistry.

Additional file 2: Figure S2. Downregulation of SRC-3 significantly inhibits the expression of IGF1, IRS1, and IRS2. The mRNA levels of IGF1, IRS1, and IRS2 were quantified by real-time PCR in stable SRC-3-knockdown and control HepG2 cells, respectively. ${ }^{*} p<0.05,{ }^{* *} p<0.01,{ }^{* * *} p<0.001$.

\section{Acknowledgements}

We thank Xiaoguang Wang for technical assistance.

\section{Authors' contributions}

$M L, Y W, C Y$ and $W L$ conceived and coordinated the study and wrote the paper. ML, YW, XX, PM, JX designed, performed and analyzed the experiments in this study. All authors reviewed the results. All authors read and approved the final manuscript.

\section{Funding}

This work was supported by grants from the Natural Science Foundation of China (No. 81772942 to C.Y), National Basic Research Program of China (973 Program, No. 2015CB553800 to C.Y), the National Science and Technology Major Project of China (2017ZX10203206 to W. L), Scientific Research Foundation for Advanced Talents, Xiang'an Hospital of Xiamen University (No. PM20180917008 to W. L), and Open Research Fund of State Key Laboratory of Cellular Stress Biology, Xiamen University (No. SKLCSB2019KF006 to X.X), Scientific Research Foundation for Doctor, Xiamen Medical College (No. K2016-12 to X.X.).

\section{Availability of data and materials}

The datasets supporting the conclusions of this article are included within the article.

Ethics approval and consent to participate

Not applicable.

\section{Consent for publication}

Not applicable.

\section{Competing interests}

The authors declare that they have no competing interests.

\section{Author details}

${ }^{1}$ Department of Hepatobiliary and Pancreatic \& Organ Transplantation Surgery, Xiang'an Hospital of Xiamen University, School of Medicine, Xiamen University, Xiamen 361012, Fujian, China. ${ }^{2}$ State Key Laboratory of Cellular Stress Biology, School of Life Sciences, Xiamen University, Xiamen 361012 , Fujian, China. ${ }^{3}$ Department of Medical Technology, Xiamen Medical College, Xiamen, China. ${ }^{4}$ Department of Molecular and Cellular Biology, Baylor College of Medicine, Houston, TX, USA.

Received: 7 May 2019 Accepted: 6 August 2019

Published online: 13 August 2019

\section{References}

1. Trepo C, Chan HLY, Lok A. Hepatitis B virus infection. Lancet. 2014;384:2053-63.

2. Yang J, Ma M, Wang XD, Jiang XJ, Zhang YY, Yang WQ, Li ZC, Wang XH, Yang B, Ma ML. Synthesis and quantitative structure-activity relationships 
study for phenylpropenamide derivatives as inhibitors of hepatitis B virus replication. Eur J Med Chem. 2015;99:82-91.

3. Arzumanyan A, Reis HM, Feitelson MA. Pathogenic mechanisms in HBV- and HCV-associated hepatocellular carcinoma. Nat Rev Cancer. 2013;13:123-35.

4. Quasdorff M, Protzer U. Control of hepatitis B virus at the level of transcription. J Viral Hepatitis. 2010;17:527-36.

5. Liang TJ. Hepatitis B: the virus and disease. Hepatology. 2009;49:S13-21.

6. Odom DT, Zizlsperger N, Gordon DB, Bell GW, Rinaldi NJ, Murray HL, Volkert TL, Schreiber J, Rolfe PA, Gifford DK, Fraenkel E, Bell GI, Young RA. Control of pancreas and liver gene expression by HNF transcription factors. Science. 2004;303:1378-81.

7. Raney AK, Johnson JL, Palmer CN, McLachlan A. Members of the nuclear receptor superfamily regulate transcription from the hepatitis $B$ virus nucleocapsid promoter. J Virol. 1997;71:1058-71.

8. Long Y, Chen E, Liu C, Huang F, Zhou T, He F, Liu L, Liu F, Tang H. The correlation of hepatocyte nuclear factor 4 alpha and 3 beta with hepatitis $B$ virus replication in the liver of chronic hepatitis B patients. J Viral Hepat. 2009; 16:537-46.

9. Reese V, Ondracek C, Rushing C, Li L, Oropeza CE, McLachlan A. Multiple nuclear receptors may regulate hepatitis $B$ virus biosynthesis during development. Int J Biochem Cell Biol. 2011;43:230-7.

10. Yu XM, Mertz JE. Distinct modes of regulation of transcription of hepatitis $B$ virus by the nuclear receptors HNF4 alpha and COUP-TF1. JVirol. 2003;77:2489-99.

11. Li L, Oropeza CE, Sainz B, Uprichard SL, Gonzalez FJ, McLachlan A. Developmental regulation of hepatitis B virus biosynthesis by hepatocyte nuclear factor 4 alpha. PloS ONE. 2009;4:e5489.

12. Liu P, Cheng H, Roberts TM, Zhao JJ. Targeting the phosphoinositide 3-kinase pathway in cancer. Nat Rev Drug Discov. 2009;8:627-44.

13. Guo H, Zhou T, Jiang D, Cuconati A, Xiao GH, Block TM, Guo JT. Regulation of hepatitis $B$ virus replication by the phosphatidylinositol 3-kinase-akt signal transduction pathway. J Virol. 2007:81:10072-80.

14. Rawat $\mathrm{S}$, Bouchard MJ. The hepatitis B virus (HBV) HBx protein activates AKT to simultaneously regulate HBV replication and hepatocyte survival. J Virol. 2015;89:999-1012.

15. Xu J, Wu RC, O'Malley BW. Normal and cancer-related functions of the p160 steroid receptor co-activator (SRC) family. Nat Rev Cancer. 2009;9:615-30.
16. Xu Y, Chen Q, Li W, Su X, Chen T, Liu Y, Zhao Y, Yu C. Overexpression of transcriptional coactivator AIB1 promotes hepatocellular carcinoma progression by enhancing cell proliferation and invasiveness. Oncogene. 2010;29:3386-97.

17. Liu YH, Tong ZW, Li T, Chen Q, Zhuo LT, Li WG, Wu RC, Yu CD. Hepatitis B virus $X$ protein stabilizes amplified in breast cancer 1 protein and cooperates with it to promote human hepatocellular carcinoma cell invasiveness. Hepatology. 2012;56:1015-24.

18. Hao J, Jin W, Li X, Wang S, Zhang X, Fan H, Li C, Chen L, Gao B, Liu G, Meng S. Inhibition of alpha interferon (IFN-alpha)-induced microRNA-122 negatively affects the anti-hepatitis B virus efficiency of IFN-alpha. J Virol. 2013:87:137-47.

19. Liu F, Song Y, Liu D. Hydrodynamics-based transfection in animals by systemic administration of plasmid DNA. Gene Ther. 1999;6:1258-66.

20. Yan J, Yu CT, Ozen M, Ittmann M, Tsai SY, Tsai MJ. Steroid receptor coactivator-3 and activator protein-1 coordinately regulate the transcription of components of the insulin-like growth factor/AKT signaling pathway. Can Res. 2006;66:11039-46.

21. Hill MM, Hemmings BA. Inhibition of protein kinase B/Akt: implications for cancer therapy. Pharmacol Ther. 2002;93:243-51.

22. Vogt PK, Bader AG, Kang S. Phosphoinositide 3-kinase: from viral oncoprotein to drug target. Virology. 2006;344:131-8.

23. Boyault S, Rickman DS, de Reynies A, Balabaud C, Rebouissou S, Jeannot E, Herault A, Saric J, Belghiti J, Franco D, Bioulac-Sage P, Laurent-Puig P, Zucman-Rossi J. Transcriptome classification of HCC is related to gene alterations and to new therapeutic targets. Hepatology. 2007;45:42-52.

24. Chandra V, Holla P, Ghosh D, Chakrabarti D, Padigaru M, Jameel S. The hepatitis E virus ORF3 protein regulates the expression of liver-specific genes by modulating localization of hepatocyte nuclear factor 4. PLoS ONE. 2011;6:e22412.

\section{Publisher's Note}

Springer Nature remains neutral with regard to jurisdictional claims in published maps and institutional affiliations.
Ready to submit your research? Choose BMC and benefit from:

- fast, convenient online submission

- thorough peer review by experienced researchers in your field

- rapid publication on acceptance

- support for research data, including large and complex data types

- gold Open Access which fosters wider collaboration and increased citations

- maximum visibility for your research: over $100 \mathrm{M}$ website views per year

At BMC, research is always in progress.

Learn more biomedcentral.com/submissions 